Journal of Animal and Veterinary Advances 10 (15): 1974-1980, 2011

ISSN: $1680-5593$

(C) Medwell Journals, 2011

\title{
The Pathogenicity Variation of Two Quail-Origin H5N1 HPAV to BALB/c Mice after Six Passages in Quail
}

\author{
${ }^{1,2}$ Hailiang Sun, ${ }^{1,2}$ Peirong Jiao, ${ }^{1,2}$ Yuqiang Cheng, ${ }^{1,2}$ Runyu Yuan, \\ ${ }^{1,2}$ Pengfei Cui, ${ }^{1,2}$ Liming Jin, ${ }^{1,2}$ Chaoan Xin and ${ }^{1,2}$ Ming Liao \\ ${ }^{1}$ Key Laboratory of Animal Disease Control and Prevention, \\ Ministry of Agriculture of P.R. China \\ ${ }^{2}$ College of Veterinary Medicine, South China Agricultural University, \\ 483 Wushan Road, 510642 Guangzhou, P.R. China
}

\begin{abstract}
H5N1 Highly Pathogenic Avian Influenza (HPAI) viruses have posed a serious threat to poultry, wild birds and mammals including humans since 1997. Quails are a potential reservoir in which influenza viruses might mutate to a mammalian transmissible form. To investigate the molecular changes that occur in H5N1 HPAI viruses following passage in quail, two isolates, A/Quail/Guangdong/342/2008 (QL342) and A/Qquail/Guangdong/176/2004 (QL176) were selected. QL342 (clade 2.3.2) and QL176 (clade 7) viruses were high pathogenic to quail with a mortality rate of $18.3-100 \%$ and could be transmitted between naive contact quails. After six passages in Japanese quails, researchers obtained two viruses, F6QL342 and F6QL176. Compared with QL342, F6QL342 had six animo acid substitutions in polymerases PB1 and PA, Nucleoprotein (NP) and Hemagglutinin (HA) but there was little difference in their pathogenicity to mice. Compared with QL176, F6QL176 virus had 10 amino acid substitutions in PB2, NA, HA proteins. F6QL176 showed an increased pathogenicity towards mice causing more severe weight loss and higher lethality compared with QL176. The findings showed that quails might play an important role in the adaptation of $\mathrm{H} 5 \mathrm{~N} 1$ avian influenza viruses to mammals. Therefore, researchers should enhance surveillance of H5N1 HPAI viruses in the quail population especially in live-bird markets.
\end{abstract}

Key words: Influenza, H5N1, BALB/c, quail, viruses, China

\section{INTRODUCTION}

H5N1 Highly Pathogenic Avian Influenza Viruses (HPAI) have the potential not only infect many species of birds including chicken, duck, quail, pigeon, mallard, bar-headed goose and sparrow but also infect mammals such as cat, dog, tiger, leopards and human (Webster et al., 1992; Lei et al., 2007; Smith et al., 2009). An H5N1 virus sample was isolated from quails sold in a live poultry retail market in Hong Kong in 2001 (Guan et al., 2002). However, the first case of quails infected with influenza A was reported in Italy as early as 1966 (Nardelli et al., 1970). Quail have the potential to support the replication of at least $14 \mathrm{HA}$ subtypes of influenza A viruses (Makarova et al., 2003). Especially in some East Asian countries where quail is farmed over a widespread area and often sold in live-bird markets, quails play an important role in the complex transmission cycles of avian influenza in live birds (Yee et al., 2009) and are considered as a source of influenza viruses that could potentially cross to humans (Shortridge et al., 1998; Webby and Webster, 2001).

For influenza viruses to accomplish interspecies transmission and expand their host range, they need to overcome selective receptor-binding mechanism. Quails possess two receptors on the epithelial cell surface in the trachea and intestine, one is a sialic acid 2,3-galactoselinked receptor to which avian viruses preferentially bind and the other is a sialic acid 2,6-galactose linked receptor to which human influenza viruses preferentially bind. (Wan and Perez, 2006). The quail therefore can be considered as an intermediate host providing an environment in which avian influenza viruses could recombine to expand their host range and possibly transmit to mammals including humans. To investigate the molecular changes in $\mathrm{H} 5 \mathrm{~N} 1$ quail-origin influenza viruses after passage in quail, researchers chose two viruses for this study and examined their selection in quail.

Corresponding Author: Ming Liao, College of Veterinary Medicine, South China Agricultural University, 483 Wushan Road, 510642 Guangzhou, People's Republic of China 
Pathogenicity to mice and the genomic variation was detected when comparing the original viruses with the passaged viruses.

\section{MATERIALS AND METHODS}

Viruses and animals: The H5N1 HPAI viruses used in this study, QL342 and QL176 were isolated from swabs taken from quails being sold in live-bird markets in Guangdong, China, in 2004 and 2008. These isolates were purified and propagated in 9-10 days old Specific-Pathogen-Free (SPF) embryonated hen eggs (Table 1). The 4 weeks old healthy Japanese quails (serological negative for avian influenza) were purchased from a quail farm in Guangzhou. The 5 weeks old SPF female $\mathrm{BALB} / \mathrm{c}$ mice were purchased from the Laboratory Animal Center of South China in Guangzhou, China and housed in BSL-3 category facilities.

Infection of quails: First, researchers examined the pathogenicity of QL342 and QL176 to quails. In each experiment, 16 quails were inoculated intranasally (i.n.) with virus in doses of $10^{6} \mathrm{Egg}$ Infectious Doses (EID) ${ }_{50}$ in a $0.1 \mathrm{~mL}$ volume. Six quails were placed in the same cage with inoculated birds to allow direct contact. At 2 Day Post Inoculation (DPI) three dead inoculated quails were dissected and the virus titers in brain, spleen, kidney, lung, colon, cloacal bursa, trachea and pancreas were measured. Tracheal and cloacal samples were collected at 3, 5, 7 and 9 DPI and tested as previous described (Perez et al., 2003). Quails were observed for mortality rate at $14 \mathrm{DPI}$.

Passages of viruses in quails: To investigate the variation of viruses in quails that occurs following passage, six quails were inoculated i.n. with virus at a dose of $10^{6} \mathrm{EID}_{50}$. As soon as possible after bird death, reisolation of the virus from lung was performed. The reisolated virus was inoculated i.n. into quails again and the virus was reisolated from the lungs of dead quails. After six passages, the virus isolates, F6QL342 and F6QL176 were obtained.

Infection of BALB/c mice: To compare the pathogenicity of progenitor virus with that of variants in mice, thirty two 5 weeks old SPF female BALB/c mice were randomly divided into four groups of eight mice each. In each group, the mice were anaesthetized by $\mathrm{CO}_{2}$ and were

Table 1: The virus used in this study

\begin{tabular}{lcc}
\hline Strain & $\mathrm{EID}_{50} / \mathrm{mL}^{\mathrm{a}}$ & $\mathrm{ELD}_{50} / \mathrm{mL}^{\mathrm{b}}$ \\
\hline A/Quail/Guangdong/342/2008 (QL342) & 8.500 & 8.32 \\
A/Quail/Guangdong/176/2004 (QL176) & 8.375 & 8.17 \\
\hline
\end{tabular}

${ }^{a}$ Virus titer expressed as $\log _{10}$; ${ }^{b}$ Lethality was determined at $48 \mathrm{~h}$ after inoculation and titers were expressed as $\log _{10}$ inoculatedi.n. withQL342, F6QL342, QL176andF6QL176 at a dose of $10^{6} \mathrm{ED}_{50}$ in a $0.05 \mathrm{~mL}$ volume, respectively. In addition, five mice were inoculated i.n. with $0.05 \mathrm{~mL}$ Phosphate Buffered Saline (PBS) as negative controls. Three mice in each group were euthanized at 2 DPI and virus titers in their brain, spleen, kidney and lung were determined. The remaining mice were checked daily for weight loss and mortality rate for 14 DPI.

Phylogenetic analysis and molecular change analysis: Viral RNA of QL342, F6QL342, QL176 and F6QL176 viruses was extracted from allantoic fluid supernatant using the RNeasy Mini Kit (Promega) following the manufacturer's instructions. RT-PCR was conducted by use of Superscript III (Invitrogen) and Uni12 (5'-AGC AAAAGCAGG-3') primer. Eight genes were amplified using universal primers, PCR products were purified with the mini PCR Purification Kit (Promega) and sequencing was performed by Shanghai Invitrogen Biotechnology Co., Ltd. Sequencing data were compiled with the Seqman program of Lasergene7 (DNASTAR). Amino acid sequence similarities were determined with the Lasergene7 Megalign program (DNASTAR). A phylogenetic tree of $\mathrm{HA}$ in the $\mathrm{H} 5 \mathrm{~N} 1$ influenza A viruses was generated using MEGA 4 software (Sinauer Associates, Inc., Sunderland, MA, USA) based on the distance-based neighbor-joining method. The HA sequences of QL342 and QL176 are available in GenBank under the relevant accession numbers (pending).

\section{RESULTS AND DISCUSSION}

Pathogenicity of H5N1 HPAI viruses in japanese quail: Both QL342 and QL176 viruses had high lethality to Japanese quails. The QL342 virus caused a 93.8\% death rate and the Mean Death Time (MDT) was 3.6 days. The mortality rate of quails inoculated with QL176 was 100\% and the MDT was 2.8 days. Two out of six contact quails died (16.7\% mortality) in both the QL342 and QL176 groups (Table 2).

QL342 and QL176 viruses could replicate well in the organs examined for the experiment. QL342 virus replicated to the mean titers of $5.2,4.37,6.78,6.7,5.95$, $4.78,5.62$ and $4.2 \log \mathrm{EID}_{50}$ in the brain, spleen, kidney, lung, colon, cloacal bursa, trachea and pancreas, respectively. QL176 virus titers in the brain, spleen,

Table 2: Mortality of experimentally inoculated quail

\begin{tabular}{lcccc}
\hline & Inoculated quail & & \multicolumn{2}{c}{ Naive contact quail } \\
& ------- & - \\
Strains & Mortality (\%) & MDT $^{\mathrm{a}}$ & Mortality (\%) & MDT \\
\hline QL342 & $93.8(15 / 16)$ & 3.6 & $33.3(2 / 6)$ & 6 \\
QL176 & $100.0(16 / 16)$ & 2.8 & $33.3(2 / 6)$ & 5 \\
\hline
\end{tabular}

${ }^{a}$ Mean death time (days post-inoculation) of dead poultry 


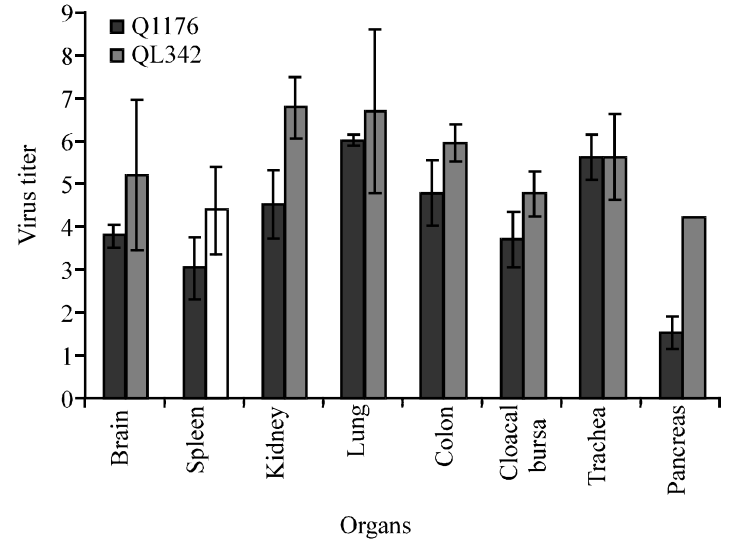

Fig. 1: Virus titers in Japanese quails at 2 days postintranasal inoculation. For statistical analysis, a value of 1.2 was assigned if the virus was detected from the undiluted sample in three embryonated hen eggs. Virus titers are expressed as means \pm standard deviation in $\log _{10} \mathrm{EID}_{50} / \mathrm{g}$ of tissue

kidney, lung, colon, cloacal bursa, trachea and pancreas were $3.78,3.03,4.53,6.03,4.78,3.7,5.62$ and $1.53 \log \mathrm{EID}_{50}$, respectively. Generally except for trachea, QL342 virus titers were a little higher than those of QL176 in the remaining organs (Fig. 1).

Viruses shedding could be detected from swabs from experimental quails before 7 DPI. QL342 virus shedding was detected at 3 DPI from oropharyngeal and cloacal swabs of inoculated quails (mean titer, 2.20 and 1.53 $\log \mathrm{EID}_{50}$, respectively). QL342 virus shedding was also detected from oropharyngeal swabs of contact quails at 3,5 and 7 DPI (mean titers, 1.62-2.20 $\log \mathrm{EID}_{50}$ ). QL342 virus was only detected in cloacal swabs of contact quails at 3 and 5 DPI (mean titers, 1. 28-1.95 $\log \mathrm{EID}_{50}$ ). QL176 virus could be detected in oropharyngeal and cloacal swabs of inoculated quails at 3 DPI (mean titers, 3.28 and $2.20 \log \mathrm{EID}_{50}$, respectively). QL176 virus could also be detected from oropharyngeal swabs of contact quails at 3,5 and 7 DPI (mean titers, 1.45-1.87 $\log \mathrm{EID}_{50}$ ). QL176 virus could only be detected in cloacal swabs of contact quails at 3 and 5 DPI (mean titers, 1.28-1.53 $\log \mathrm{EID}_{50}$ ). Neither of two viruses was detected from swabs of inoculated or naive contact quails at 9 DPI (Table 3 ).

Pathogenicity in BALB/c mice: To detect any difference in pathogenicity between the original virus isolates and viruses passaged in mice, $\mathrm{SPF}$ female $\mathrm{BALB} / \mathrm{c}$ mice were inoculated i.n. at a dose of $10^{6} \mathrm{EID}_{50}$ in $0.05 \mathrm{~mL}$. All mice exposed to the QL342 virus died by 7 DPI while those inoculated with the F6QL342 virus died by 6 DPI. The mortality rate of mice inoculated with QL176 was $20 \%$ while the mortality rate of mice inoculated with F6QL176 was 60\% (Fig. 2). QL342 virus replicated systemically to
Table 3: Virus titers in cloacal or oropharyngeal swabs from quails Days post inoculation

3 5

Strains Oro-swab ${ }^{c}$ Clo-swab $^{\mathrm{d}}$ Oro-swab Clo-swab Oro-swab Clo-swab $\mathrm{A} / \mathrm{Ql} / \mathrm{GD} / \mathbf{3 4 2} / 08$

$\begin{array}{lllllllllllll}\mathrm{I}^{\mathrm{a}} & 3 / 3^{\mathrm{c}} & 2.20^{\mathrm{d}} & 2 / 3 & 1.53 & 1 / 1 & \mathrm{ND}^{\mathrm{e}} & 0 / 1 & \mathrm{ND} & 0 / 1 & \mathrm{ND} & 0 / 1 & \mathrm{ND}\end{array}$ $\begin{array}{lllllllllllll}\mathrm{C}^{\mathrm{b}} & 2 / 3 & 1.62 & 1 / 3 & 1.28 & 2 / 3 & 2.20 & 2 / 3 & 1.95 & 1 / 3 & 1.87 & 3 / 3 & \mathrm{ND}\end{array}$ $\mathrm{A} / \mathrm{Ql} / \mathrm{GD} / \mathbf{1 7 6} / \mathbf{0 4}$

$\begin{array}{llllll}\text { I } & 3 / 3 & 3.28 & 3 / 3 & 2.20 & -8\end{array}$

$\begin{array}{llllllllllllll}\mathrm{C} & & 1 / 3 & 1.45 & 1 / 3 & 1.53 & 3 / 3 & 1.87 & 1 / 3 & 1.28 & 2 / 3 & 1.70 & 3 / 3 & \mathrm{ND}\end{array}$

For statistical purposes, a value of 1.2 was assigned if virus was not detected from the undiluted sample in three embryonated hen's eggs; "Quails

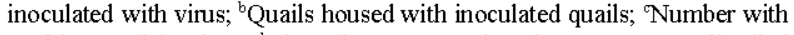
positive swab/total N; ${ }^{d}$ Virus titer expressed as $\log _{10} \mathrm{EDD}_{50}$; ${ }^{e}$ Quails died before 5 day post-inoculation; Oro-swab: Oropharyngeal swabs; Clo-swab: Cloacal swabs; ND: Not Detected

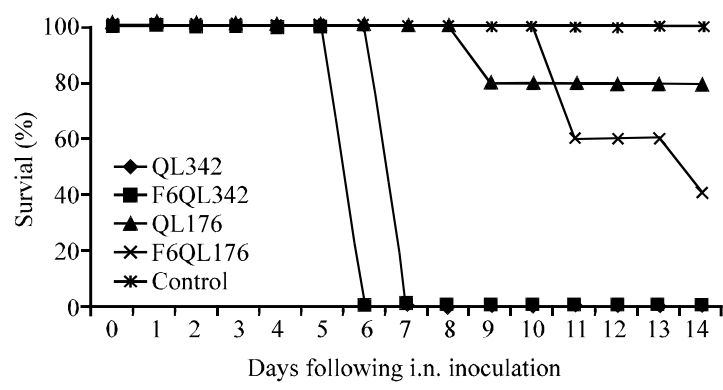

Fig. 2: Lethality of the QL342, F6QL342, QL176 and F6QL176 viruses to mice. Survival curves of the mice inoculated with different $\mathrm{H} 5 \mathrm{~N} 1$ viruses, QL342, F6QL342, QL176 and F6QL176 with the doses of $10^{6} \mathrm{EID}_{50}$

a titer of $6.13 \log \mathrm{EID}_{50}$ in the lung and to $2.17,1.34$ and $1.08 \log \mathrm{EID}_{50}$ in the spleen, kidney and brain, respectively. F6QL342 virus replicated to a mean titer of $6.75 \log \mathrm{EID}_{50}$ in the lung and to $2.55,1.58$ and $1.08 \log \mathrm{EID}_{50}$ in the spleen, kidney and brain, respectively. QL176 virus replicated systemically to a titer of 3.34 and $1.04 \log \mathrm{EID}_{50}$ in the lung and spleen. F6QL176 virus replicated to a mean titer of 4.08 and $1.33 \operatorname{logEID}{ }_{50}$ in the lung and spleen (Fig. 3). Infection with QL176 virus caused a 7.17\% body weight loss by 14 DPI. F6QL176 virus caused an $11.05 \%$ body weight loss by 14 DPI (Fig. 4). In all, the pathogenicity of passaged viruses was greater than that of the original virus isolates.

Molecular change analysis between original virus Isolates and passaged viruses: The virus RNA was sequenced in order to detect any molecular differences between original virus isolates and passaged viruses. When comparing QL342 to the passaged F6QL342, six amino acids substitutions were found: V451I and C730Y in polymerase $\mathrm{PB} 1 ; \mathrm{N} 228 \mathrm{I}$ in polymerase PA; D101E in Nucleoprotein (NP) and Q376L and N497S in 


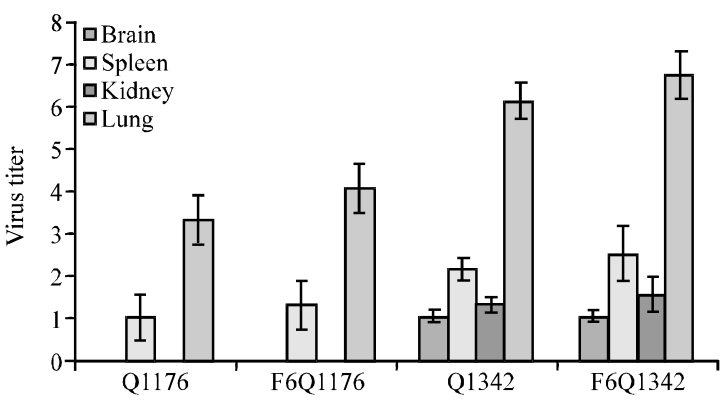

Fig. 3: Virus titers of organs of BALB/c mice at 3 days post-intranasal inoculation. For statistical analysis, a value of 1 was assigned if the virus was not detected from the undiluted sample in three embryonated hen eggs. Virus titers are expressed as means \pm standard deviation in $\log _{10} \mathrm{EID}_{50} /$ gram of tissue

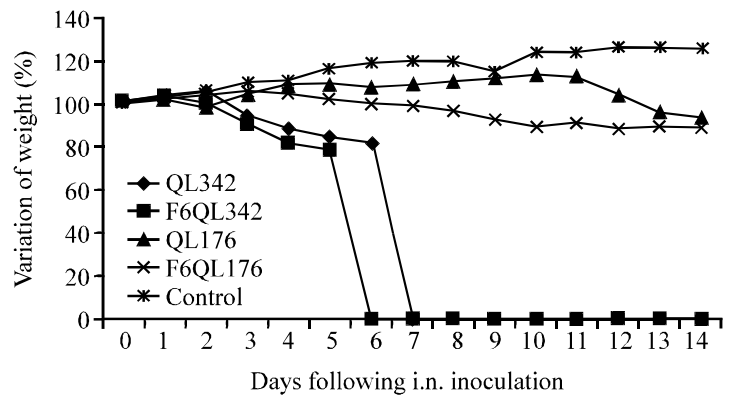

Fig. 4: Weight variation of BALB/c mouse during the 14 days post-inoculation. The mean weight variation of the mice inoculated with different $\mathrm{H} 5 \mathrm{~N} 1$ viruses, QL342, F6QL342, QL176 and F6QL176 at a dose of $10^{6} \mathrm{ED}_{50}$

Table 4: The amino acids substitution between QL342 and F6QL342 Amino acid substitution

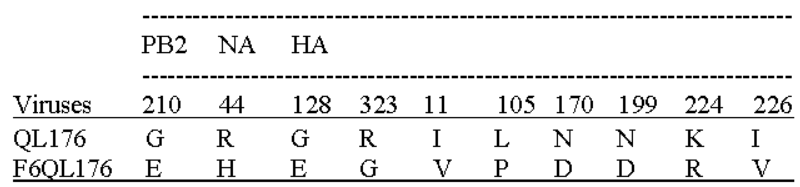

Amino acids sites were located by taking $\mathrm{A} / \mathrm{Gs} / \mathrm{GD} / 1 / 96$ as reference

Hemagglutinin (HA) (Table 4). There were 10 amino acids changes between QL176 and passaged F6QL176: G201E in polymerase $\mathrm{PB} 2$; $\mathrm{R} 44 \mathrm{H}, \mathrm{G} 128 \mathrm{E}$ and $\mathrm{R} 323 \mathrm{G}$ in Neuraminidase (NA) and I11V, L105P, N170D, N199D, $\mathrm{K} 224 \mathrm{R}$ and $\mathrm{I} 226 \mathrm{~V}$ in $\mathrm{HA}$.

The HA phylogenetic analysis of quail-origin H5N1 virus: To better understand the characteristics of the HA antigen and the degree of circulation of H5N1 viruses found in the quail population, the $\mathrm{HA}$ sequences from domestic poultry, wild birds, humans and quails available in GenBank were used to construct an HA phylogenetic tree. Quail-origin H5N1 influenza viruses isolated in some countries of southeast Asia during 2001-2004 were mainly the viruses classified as clades $1,2.1 .1,2.1 .3,3,5$ and 7 . Viruses classified as clades $1,2.2$ and 2.3 .2 were the primary $\mathrm{H} 5 \mathrm{~N} 1$ viruses found to be circulating in quails in the years 2005-2008. H5N1 influenza viruses isolated from quails in Thailand or Viet Nam between 2004 and 2005 were clade 1 viruses. Quail-origin $\mathrm{H} 5 \mathrm{~N} 1$ influenza viruses isolated in China during 2001-2008 were mainly clade 1 , 2.3.2, 3, 5 and 7 viruses. QL342 could be classified as belonging to clade 2.3.2 viruses while QL176 was member of the clade 7 virus group (Fig. 5).

Since 2003, H5N1 HPAI viruses have caused several disease outbreaks in many countries in Asia, Europe and Africa that have resulted in large economic losses and posed serious threats to public health. H5N1 HPAI circulating in quails has been reported in several Asian countries including Indonesia, Viet Nam, Korea, Egypt and China. The viruses isolated from quails in these countries mainly belonged to the clade $1,2.1 .1,2.1 .3,2.2$, 2.3.2, 2.3.4, 5 and 7 virus groups. In addition, clades 2.3 .2 and 7 viruses were found to be the predominant group circulated in poultry and wild birds in China during 2007-2009 (Jiang et al., 2010). Clade 2.3.2 and 7 H5N1 viruses in particular had the potential to infect humans. (Xu et al., 2009). In the study, QL342 was characterised as a member of the clade 2.3.2 viruses while QL176 belonged to the clade 7 virus group therefore researchers selected these two viruses to passage in quails (Table 5).

Previous studies have shown that quails are very susceptible to infection with H5N1 HPAI viruses and have a high mortality rate and that these viruses are able to replicate systemically in brain, spleen, kidney, lung, trachea, intestine, heart, liver and skeletal muscle (Perkins and Swayne, 2001; Saito et al., 2009; Antarasena et al., 2006). The findings showed that QL342 and QL176 virus isolates were highly pathogenic to Japanese quails when inoculated with $10^{6} \mathrm{EID}_{50} / 0.05 \mathrm{~mL}$ with a MDT of 3.6 days . The two viruses replicated to very high virus titers in the brain, spleen, kidney, lung, colon, cloacal bursa, trachea and pancreas. In addition, in quail it has previously been recognised that influenza viruses could be shed via the respiratory tract rather than just in faeces (Liu et al., 2003). Researchers found that the titers of virus in oropharyngeal swabs were higher than those isolated from cloacal swabs.

Although, in mice the 2,3-linked Sialic Acid (SA) receptor is distributed in ciliated airways and in particular on type II alveolar epithelial cells, the 2,6-linked SA 


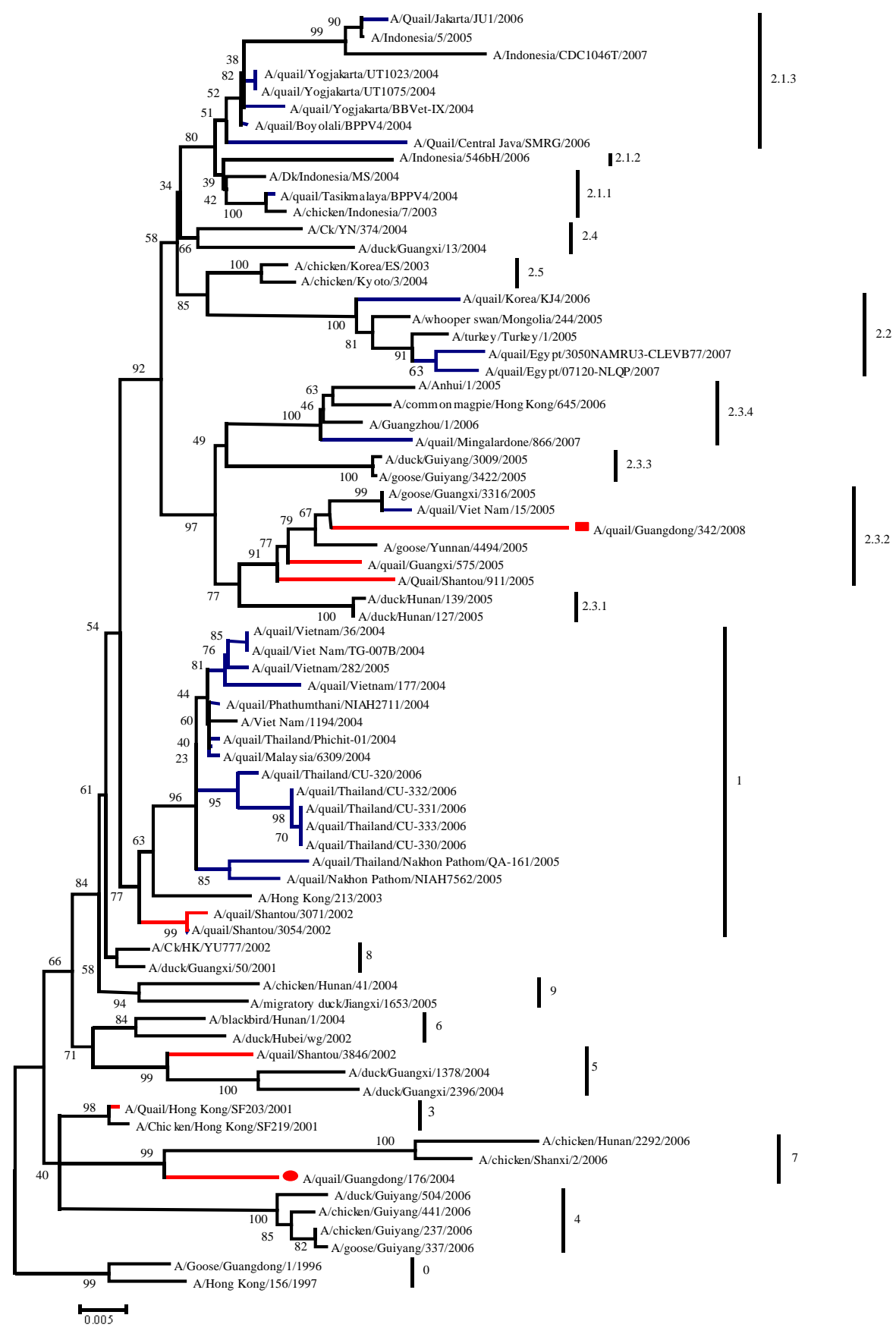

Fig. 5: Phylogenetic tree of HA in the H5N1 influenza A viruses. The HA tree was generated with MEGA 4 software based on nucleotides (nt) 29-1732 using the distance-based neighbor-joining method. The reliability of the tree was assessed by bootstrap analysis with 1000 replicates. Horizontal distances are proportional to genetic distance. Except for the two isolates, other virus sequences were downloaded from GenBank

receptor has not been found to be expressed (Ibricevic et al., 2006). Despite this, mice are often used as a convenient small animal model to determine the pathogenicity of different avian influenza viruses in mammalian species (Chen et al., 2004; Jiao et al., 2008). $\mathrm{H} 5 \mathrm{~N} 1$ viruses are able to infect mice without adaptation and manifest different levels of lethality in different strains (Chen et al., 2004; Jiao et a l., 2008). In the study, there 
Table 5: The amino acids substitution between QL176 and F6QL176 Amino acid substitution

\begin{tabular}{lllllll} 
& PB1 & & PA & NP & HA & \\
& - & & - & - \\
Viruses & 451 & 730 & 228 & 101 & 376 & 497 \\
\hline QL342 & V & C & N & D & Q & N \\
F6QL342 & I & Y & I & E & L & S \\
\hline
\end{tabular}

Amino acids sites were located by taking $\mathrm{A} / \mathrm{Gs} / \mathrm{GD} / 1 / 96$ as reference

was a $100 \%$ lethality rate in $\mathrm{BALB} / \mathrm{c}$ mice inoculated with a dose of $10^{6} \mathrm{ED}_{50} \mathrm{H} 5 \mathrm{~N} 1 \mathrm{QL} 342$ virus in a $0.05 \mathrm{~mL}$ volume and this virus could spread systemically to replicate in brain, spleen, kidney and lung. QL176 virus had low pathogenicity to $\mathrm{BALB} / \mathrm{c}$ mice given a dose of $10^{6} \mathrm{EID}_{50}$. The lethality rate of QL176 to mice was $20 \%$ and the virus only replicated in the spleen and the lung. After six passages in the lung of quails, F6QL342 virus also had $100 \%$ lethality to mice and its pathogenicity was a little higher than that of QL176. In contrast, F6Q1176 manifested an increased pathogenicity to mice, causing more severe weight loss and possessing stronger replication capability and higher lethality compared with Q1176.

Quails possess two receptors, 2,3-galactose-linked receptors and 2,6-galactose-linked receptors on the epithelial cells of their trachea and intestine. It was found previously that the $H A$ gene of some H5N1 viruses with human-type receptor specificity reverted back to only recognizing avian-type receptors following passage in duck (Shinya et al., 2010).

\section{CONCLUSION}

This study suggested that avian influenza virus receptor-linked specificity could change by passages in the host in vivo. The researchers want to clarify if QL342 or QL176 had gained human receptor-linked specificity following passage in quails. However, the study showed that the amino acids present in the HA receptor-binding sites of the two viruses were not changed after six passages in quails. Interestingly, the substitution N170D in $\mathrm{HA}$ protein in the $\mathrm{F} 6 \mathrm{Q} 1176$ isolate resulted in the absence of a potential N-linked glycosylation site. Glycosylation can affect hemagglutinin receptor affinity and also the efficiency of release of new virus from host cells (Gallagher et al., 1992; Abe et al., 2004). There were nine animo acids changes in NA and HA proteins from the F6Q1176 isolate that could potentially change the virus membrane characteristics.

\section{RECOMMENDATIONS}

Further studies using reverse genetics are needed to confirm if the changes in HA and NA sequence found following passage in quails are able to enhance the pathogenicity of the F6Q1176 isolate in mice.

\section{ACKNOWLEDGEMENTS}

This research was supported by grants from the Natural Science Foundation of Guangdong province (No. 10251064201000004 , No. 10151064201000021 and NO. 5200638), the Program for Changjiang Scholars and Innovative Research Team in Chinese Universities (No. IRT0723) and the Science and Technology Projects of Guangdong province (No. 20100206). Promgram for National Broiler Industry (nycytx-42-G3-03). Promgram for Healthy Breeding Technology Research (2009A0201006). Innovative Research Team in University (No. IRT0723), the Science and Technology Projects of Guangdong province (No. 20100206 and 2010B020307005) and highlevel talents in university project of Guangdong province.

\section{REFERENCES}

Abe, Y., E. Takashita, K. Sugawara, Y. Matsuzaki, Y. Muraki and S. Hongo, 2004. Effect of the addition of oligosaccharides on the biological activities and antigenicity of influenza $\mathrm{A} / \mathrm{H} 3 \mathrm{~N} 2$ virus hemagglutinin. J. Virol., 78: 9605-9611.

Antarasena, C., R. Sirimujalin, P. Prommuang, S.D. Blacksell, N. Promkuntod and P. Prommuang, 2006. Tissue tropism of a Thailand strain of highpathogenicity avian influenza virus (H5N1) in tissues of naturally infected native chickens (Gallus gallus), Japanese quail (Coturnix coturnix japonica) and ducks (Anas spp.). Avian Pathol., 35: 250-253.

Chen, H., G. Deng, Z. Li, G. Tian and Y. Li et al., 2004. The evolution of H5N1 influenza viruses in ducks in southern China. Proc. Natl. Acad. Sci. USA., 101: 10452-10457.

Gallagher P.J., J.M. Henneberry, J.F. Sambrook and M.J. Gething, 1992. Glycosylation requirements for intracellular transport and function of the hemagglutinin of influenza virus. J. Virol., 66: 71367145.

Guan, Y., J.S.M. Peiris, A.S. Lipatov, T.M. Ellis and K.C. Dyrting et al., 2002. Emergence of multiple genotypes of H5N1 avian influenza viruses in Hong Kong SAR. Proc. Natl. Acad. Sci., 99: 8950-8955.

Ibricevic, A., A. Pekosz, M.J. Walter, C. Newby and J.T. Battaile et al., 2006. Influenza virus receptor specificity and cell tropism in mouse and human airway epithelial cells. J. Virol., 80: 7469-7480.

Jiang, W.M., S. Liu, J. Chen, G.Y. Hou and J.P. Li et al., 2010. Molecular epidemiological surveys of H5 subtype highly pathogenic avian influenza viruses in poultry in China during 2007-2009. J. Gen. Virol., 91: 2491-2496. 
Jiao, P.R., G.B. Tian, Y.B. Li, G.H. Deng and Y.P. Jiang et al., 2008. A single-amino-acid substitution in the NS1 protein changes the pathogenicity of $\mathrm{H} 5 \mathrm{N1}$ avian influenza viruses in mice. J. Virol., 82: 1146-1154.

Lei, F., S. Tang, D. Zhao, X. Zhang and Z. Kou et al., 2007. Characterization of H5N1 influenza viruses isolated from migratory birds in Qinghai province of China in 2006. Avian Dis., 51: 568-572.

Liu, M., S. He, D. Walker, N. Zhou and D.R. Perez et al., 2003. The influenza virus gene pool in a poultry market in South Central China. Virology, 305: 267-275.

Makarova, N.V., H. Ozaki, H. Kida andR.G. Webster, 2003. Replication and transmission of influenza viruses in Japanese quail. Virology, 310: 8-15.

Nardelli, L., A. Rinaldi, H.G. Pereira and G. Mandelli, 1970. Influenza virus infections in Japanese quails. Arch. Exp. Veterinarmed, 24: 231-249.

Perez, D.R. W. Lim, J.P. Seiler, G. Yi, M. Peiris, K.F. Shortridge and R.G. Webster, 2003. Role of quail in the interspecies transmission of $\mathrm{H} 9$ influenza a viruses: Molecular changes on HA that correspond to adaptation from ducks to chickens. J. Virol., 77: $3148-3156$.

Perkins and Swayne, 2001. Pathobiology of A/chicken/Hong Kong/220/97 (H5N1) avian influenza virus in seven gallinaceous species. Vet. Pathol., 38: 149-164.

Saito, T., C. Watanabe, N. Takemae, A. Chaisingh and Y. Uchida et al., 2009. Pathogenicity of highly pathogenic avian influenza viruses of H5N1 subtype isolated in Thailand for different poultry species. Vet. Microbiol., 133: 65-74.
Shinya, K., A. Makino, M. Hatta, S. Watanabe, J.H. Kim and Y. Kawaoka, 2010. A mutation in H5 haemagglutinin that conferred human receptor recognition is not maintained stably during duck passage. J. Gen Virol., 91: 1461-1463.

Shortridge, K.F., N.N. Zhou, Y. Guan, P. Gao and T. Ito et al., 1998. Characterization of avian H5N1 influenza viruses from poultry in Hong Kong. Virology, 252: 331-342.

Smith, G.J., D. Vijaykrishna, T.M. Ellis, K.C. Dyrting and Y.H. Leung et al., 2009. Characterization of avian influenza viruses A (H5N1) from wild birds, Hong Kong, 2004-2008. Emerging Infectious Dis., 1: 402-407.

Wan, H. and D.R. Perez, 2006. Quail carry sialic acid receptors compatible with binding of avian and human influenza viruses. Virology, 346: 278-286.

Webby, R.J. and R.G. Webster, 2001. Emergence of influenza a viruses. Philos. Trans. R. Soc. Lond. B Biol. Sci., 356: 1817-1828.

Webster, R.G., M. Yakhno, V.S. Hinshaw, O.T. Gorman and Y. Kawaoka, 1992. Evolution and ecology of influenza a viruses. Microbiol. Rev., 56: 152-179.

Xu, C., L. Dong, L. Xin, Y. Lan, Y. Chen, L. Yang and Y. Shu, 2009. Human avian influenza A (H5N1) virus infection in China. Life Sci., 52: 407-411.

Yee, K.S., C.J. Cardona and T. E. Carpenter, 2009. Transmission of low-pathogenicity avian influenza virus of subtype HøN2 from chickens to Pekin ducks and Japanese quail (Coturnix coturnix japonica). Avian Pathol., 38: 59-64. 